\title{
VISCOUS COSMOLOGIES WITH EXTRA DIMENSIONS
}

\author{
B. C. Paul* \\ Physics Department, North Bengal University, \\ Siliguri, Dist. Darjeeling, PIN 734 430, India
}

\begin{abstract}
We present an analysis of a n-dimensional vacuum Einstein field equations in which 4-dimensional space-time with is described by a Friedmann Robertson-Walker (FRW) metric and that of the extra dimensions by a Kasner type Euclidean metric. The field equations are interpreted as four dimensional Einstein equations with effective matter properties. We obtain solutions for the cases when the effective matter is described by imperfect fluid. We consider the theories of imperfect fluid given by Eckart, truncated Israel-Stewart and full Israel-Stewart theories and obtain cosmological solutions for a flat model of the universe.
\end{abstract}

PACS numbers : $04.20 \mathrm{Jb}, 98.80 \mathrm{H}$

*Electronic mail : bcpaul@nbu.ernet.in 


\section{INTRODUCTION :}

Model building in higher dimensions was initiated by Kaluza and Klein ${ }^{1}$ who tried to unify gravity with electromagnetic interaction by introducing an extra dimension. Kaluza-Klein theory is basically an extension of Einstein general relativity in 5D which is of much interest in Particle Physics and Cosmology. In the last few decades the study of a higher dimensional theory has been revived and considerably generalized after realizing that many interesting theories of particle interactions need more than four dimensions for their formulation. Attempts have been made to build cosmological models ${ }^{2}$ in higher dimensions which may undergo a spontaneous compactification leading to a product space $M^{4} \times M^{d}$, with $M^{d}$ describing the compact innerspace. In the usual approach one uses Einstein's field equation

$$
G_{a b}=8 \pi G T_{a b}
$$

which match the Einstein tensor and the energy momentum tensor. Recently another approach ${ }^{3}$ has been developed taking $G_{a b}=0$, the extra terms which appear in these equations due to extra dimensions are interpreted as induced or effective properties of matter in ordinary 4D space-time. Ibánez and Verdaguer [henceforth IV $]^{4}$ first obtained a set of solutions of Einstein's equation in an n-dimensional vacuum. They found homogeneous solutions with expanding three dimensional isotropic space. The solutions are then identified with the observable four dimensional subspace with perfect fluid. Later a class of solutions in flat universe model have been obtained by Gleiser and Diaz. ${ }^{5}$ Krori et al ${ }^{6}$ using a higher dimensional anisotropic cosmology (Bianchi-I) obtained 4D perfect fluid solutions. It was shown that the perfect fluid solutions obtained by them are compatible with contraction of all the extra 
dimensions. The solutions are identified with a 4 dimensional anisotropic cosmological model. However, it is known that the matter distribution in the early universe may not be as simple as predicted by perfect fluid due to a number of dissipative processes. Hence it is essential to study cosmological solutions in the framework of imperfect fluid description. The simple theory is the Eckart theory, ${ }^{7}$ however, it suffers from serious drawbacks concerning causality and stability. ${ }^{8,9}$ These difficulties can, however, be removed by considering higher order theories i.e., extended irreversible thermodynamics (EIT). ${ }^{10,11}$ In this paper we intend to study a higher dimensional vacuum Einstein equations which leads to an observed 4 dimensional universe with imperfect fluid. We obtain exact solutions in the framework of the Eckart, truncated Israel-Stewart (TIS) and full Israel-Stewart (FIS) theories.

The plan of the paper is as follows : In sec. 2 we set up the relevant field equations, in sec. 3 cosmological solutions are presented and finally in sec 4 we discuss the results.

\section{FIELD EQUATIONS :}

We consider a higher dimensional metric in the form

$$
d s^{2}=d s_{F R W}^{2}+\sum_{i=1}^{d} b_{i}^{2}(t) d x_{i}^{2}
$$

where $d$ is the number of extra dimensions $(d=n-4)$ and $d s_{F R W}^{2}$ represents the line element of the FRW metric in four dimensions which is given by

$$
d s^{2}=-d t^{2}+a^{2}(t)\left[\frac{d r^{2}}{1-k r^{2}}+d \Omega^{2}\right]
$$


where $a(t)$ is the scale factor of the 4 dimensional spacetime and $b_{i}$ 's are the scale factors for the extra dimensions and $k=0,+1,-1$ represents flat, closed and open universe respectively.

The vacuum Einstein's equation in n-dimensions using the metric (2) can be written as

$$
\begin{aligned}
& 3\left(\frac{\dot{a}^{2}+k}{a^{2}}\right)=\frac{\ddot{D}}{D}-\frac{1}{8}\left(\sum_{i=1}^{d} \frac{{\dot{b_{i}}}^{2}}{b_{i}^{2}}\right)^{2}-\frac{1}{8} \sum_{i=1}^{d}\left(\frac{{\dot{b_{i}}}^{2}}{b_{i}^{2}}\right)^{2}, \\
& 2 \frac{\ddot{a}}{a}+\frac{\dot{a}^{2}+k}{a^{2}}=\frac{\dot{a} \dot{D}}{a D}+\frac{1}{8}\left(\sum_{i=1}^{d} \frac{\dot{b}_{i}{ }^{2}}{b_{i}^{2}}\right)^{2}-\frac{1}{8} \sum_{i=1}^{d}\left(\frac{\dot{b}_{i}{ }^{2}}{b_{i}^{2}}\right)^{2}, \\
& \frac{\ddot{b}_{i}}{b_{i}}+3 \frac{\dot{a} \dot{b}_{i}}{a b_{i}}+\frac{\dot{D} b_{i}}{D b_{i}}-\frac{\dot{b}_{i}^{2}}{b_{i}^{2}}=0
\end{aligned}
$$

where we denote

$$
D^{2}=\Pi_{i=1}^{d} b_{i}(t)
$$

and set $8 \pi G=1$. Using eq.(7) in eq.(6) one gets

$$
\frac{\ddot{D}}{D}=-3 \frac{\dot{a} \dot{D}}{a D}
$$

On integrating eq. (8), one obtains

$$
\dot{D} a^{3}=C
$$

where $C$ is an integration constant. Using eqs.(6) and (9) one gets

$$
b_{i}=D^{2 p_{i}}
$$

with $\sum_{i=1}^{d} p_{i}=1$. Using eq.(10) the field eqs.(4)-(5) can be rewritten as

$$
3\left(\frac{\dot{a}^{2}+k}{a^{2}}\right)=\frac{\ddot{D}}{D}+\alpha\left(\frac{\dot{D}}{D}\right)^{2},
$$




$$
-\left(2 \frac{\ddot{a}}{a}+\frac{\dot{a}^{2}+k}{a^{2}}\right)=-\frac{\dot{a} \dot{D}}{a D}+\alpha\left(\frac{\dot{D}}{D}\right)^{2}
$$

where $\alpha=\frac{1}{2}\left[\sum_{i=1}^{d} p_{i}^{2}-1\right]$. We now use eqs.(9), (11) and (12) to obtain cosmological models. Eqs. (11) and (12) may be identified as the Einstein field equations in 4D when one equates the left hand side with energy density $(\rho)$ and pressure $(\mathrm{p})$ respectively. The Einstein field equation with imperfect fluid in 4-dimensions is represented by

$$
\begin{gathered}
3\left(\frac{\dot{a}^{2}+k}{a^{2}}\right)=\rho \\
2 \frac{\ddot{a}}{a}+\frac{\dot{a}^{2}+k}{a^{2}}=-P_{e f f}
\end{gathered}
$$

where $P_{\text {eff }}=p+\Pi, \Pi$ is the bulk viscous stress and $\mathrm{p}$ is the thermodynamic pressure which is related to density as $p=(\gamma-1) \rho(1<\gamma<2)$.

We now compare eqs.(11) and (12) with that of eqs.(13) and (14) and identified the effective energy density and pressure in terms of higher dimensional scale factors which are

$$
\rho=\frac{\ddot{D}}{D}+\alpha\left(\frac{\dot{D}}{D}\right)^{2}
$$

and

$$
p+\Pi=-\frac{\dot{a} \dot{D}}{a D}+\alpha\left(\frac{\dot{D}}{D}\right)^{2}
$$

Using the relation for energy density and pressure, we determine the bulk viscous stress from eqs. (15) and (16) which is given by

$$
\Pi=-\left(\gamma-\frac{4}{3}\right) \frac{\ddot{D}}{D}-\alpha(\gamma-2)\left(\frac{\dot{D}}{D}\right)^{2}
$$

It is evident here that for $\alpha=0$ and $\gamma=\frac{4}{3}$ it leads to cosmological solutions with radiation i.e. without viscosity which was studied by IV in the context of perfect 
fluid in 4-dimensions. We study here cosmological solutions for $\alpha \neq 0$, which leads to interesting results. The solutions are identified with a $4 \mathrm{D}$ universe with imperfect fluid. The evolution of the bulk viscous stress in $(3+1)$ dimensions is given by ${ }^{10}$

$$
\Pi+\tau \dot{\Pi}=-3 \zeta H-\frac{\epsilon}{2} \tau \Pi\left(3 H+\frac{\dot{\tau}}{\tau}-\frac{\dot{\zeta}}{\zeta}-\frac{\dot{T}}{T}\right)
$$

where $\zeta$ is the coefficient of bulk viscosity, $\tau$ is the relaxation time and $T$ is the temperature and $\mathrm{H}\left(\frac{\dot{a}}{a}\right)$

is the Hubble parameter. Here the parameter $\epsilon$ can take the value 0 or $1, \epsilon=0$ for TIS and $\epsilon=1$ for FIS theory. One obtains Eckart theory for $\tau=0$. The system of eqs. (11), (15), (17) and (18) are not closed as number of unknowns are more than the number of equations. It is, therefore, necessary to assume the following adhoc but commonly chosen relations

$$
\zeta=\beta \rho^{q} \text { and } \tau=\beta \rho^{q-1}
$$

where $q \geq 0$. Using eq.(18) one obtains the behavior of temperature $T$ for a universe with imperfect fluid.

\section{COSMOLOGICAL SOLUTIONS :}

Let us consider a power law model of the expansion of the universe given by

$$
a=a_{o} t^{m}
$$

where $a_{o}$ and $\mathrm{m}$ are constants. The energy density is given by

$$
\rho=\frac{3 m^{2}}{t^{2}}
$$

for a flat universe $(k=0)$. 
We now obtain solutions for Eckart, TIS and FIS theories :

Case I : Eckart theory :

It corresponds to $\tau=0$ and we get

$$
\Pi=-3 \zeta H
$$

For $q=\frac{1}{2}$ i.e., $\zeta=\beta \rho^{1 / 2}$ we consider two values for $\gamma$.

(i) when $\gamma=\frac{4}{3}$ one gets power law expansion for $\beta=\frac{2(2 m-1)}{\sqrt{3} m}$ for $m>\frac{1}{2}$. A large power law inflation $(m \rightarrow \infty)$ may be obtained when $\beta \rightarrow \frac{4}{\sqrt{3}}$.

(ii) when $\gamma=2$, we obtain $\beta=\frac{2(3 m-1)}{3 \sqrt{3} m}$. One obtains huge power law inflation for $\beta \rightarrow \frac{2}{\sqrt{3}}$

Case II : TIS theory :

It corresponds to $\epsilon=0$. Eq.(18) for the bulk viscous stress (П) reduces to

$$
\Pi+\tau \dot{\Pi}=-3 \zeta H
$$

The solution of the above equation may be obtained for different values of $\gamma$ a parameter in the equation of state. We consider two different cases :

(i) For $\gamma=\frac{4}{3}$ i.e. radiation, one gets the bulk viscous stress which is given by

$$
\Pi=\frac{2 \alpha(1-3 m)^{2}}{3 t^{2}}
$$

It depends both on $\alpha$ and on the exponent $m$. Here $\alpha$ can be evaluated knowing eq.(19). For simplicity consider a case $q=\frac{1}{2}$ and we get

$$
\beta=\frac{2 \sqrt{3}(2 m-1) m}{9 m^{2}+8 m-4}
$$

In this scenario one gets physically relevant solution for $m>\frac{1}{2}$. 
(ii) For $\gamma=2$ i.e. stiff fluid, one gets the bulk viscous stress given by

$$
\Pi=-\frac{2 m(3 m-1)}{t^{2}}
$$

which is negative for $m \geq \frac{1}{3}$. In this case we note that the bulk viscous stress is independent of $\alpha$. in this case. For $q=\frac{1}{2}$, using eq. (22) we get $\beta=\frac{2 \sqrt{3} m(3 m-1)}{9 m^{2}+12 m-4}$. For a realistic scenario $\beta>0$ which is obtained for $m>\frac{1}{3}$.

Case III : FIS theory :

In this case we put $\epsilon=1$ and eq.(18) reduces to

$$
\Pi+\tau \dot{\Pi}=-3 \zeta H-\frac{1}{2} \tau \Pi\left(3 H+\frac{\dot{\tau}}{\tau}-\frac{\dot{\zeta}}{\zeta}-\frac{\dot{T}}{T}\right)
$$

(i) For radiation $\left(\gamma=\frac{4}{3}\right)$, the bulk viscous stress is determined from eq.(17) is given by

$$
\Pi=\frac{2 \alpha}{3} \frac{(1-3 m)^{2}}{t^{2}}
$$

Using $q=\frac{1}{2}$ for relations (19) we obtain the behaviour of temperature from eq.(26) which takes the form given by

$$
T=C_{1} t^{a_{1}}
$$

where $a_{1}=3 m-4+\frac{2 \sqrt{3} m}{\beta}+\frac{27 m^{3}}{\alpha(1-3 m)^{2}}$. One gets a decreasing mode of temperature in this regime for $\beta>\frac{2 \sqrt{3} m(2 m-1)}{3 m^{2}+11 m-4}$, which determines the relaxation time and coefficient of viscosity. In this case one gets solution for $m>\frac{1}{2}$. However, at $m=\frac{1}{2}$ one gets a non-viscous universe.

(ii) For a stiff fluid ( $\gamma=2$ ), the bulk viscous stress is given by

$$
\Pi=-\frac{2 m(3 m-1)}{t^{2}}
$$


which is independent of $\alpha$. Here $\Pi<0$ is obtained for $m>\frac{1}{3}$. Using $q=\frac{1}{2}$ in eq. (19) we obtain the behavior of temperature of the universe which is given by

$$
T=C_{2} t^{a_{2}}
$$

where $a_{2}=\frac{4-15 m}{3 m-1}+\frac{2 \sqrt{3} m}{\beta}$. One gets a decreasing mode of temperature in this regime for $\beta>\frac{2 \sqrt{3} m(3 m-1)}{15 m-4}$, which admits a power law expansion with exponent $m$ having values $m>\frac{1}{3}$. The table given below shows a comparative values of $\beta$ for $\gamma=\frac{4}{3}$ and $\beta^{\prime}$ for $\gamma=2$ i.e., the co-efficient of viscosity for a given type of expansion of the universe :

\begin{tabular}{||c|c|c|c|c|c|c||}
\hline \multicolumn{7}{||c}{ variation of $\beta$ in Ekart, TIS, FIS theories } \\
\hline$m$ & $\beta_{\text {Eckart }}$ & $\beta_{\text {TIS }}$ & $\beta_{\text {FIS }}$ & $\beta_{\text {Eckart }}^{\prime}$ & $\beta_{\text {TIS }}^{\prime}$ & $\beta_{F I S}^{\prime}$ \\
\hline 0.5 & 0 & 0 & $>0$ & 0.3849 & 0.2038 & $>0.2474$ \\
\hline 0.6 & 0.3849 & 0.1028 & $>0.1136$ & 0.5132 & 0.2582 & $>0.3326$ \\
\hline 0.7 & 0.6598 & 0.1614 & $>0.1876$ & 0.6048 & 0.3028 & $>0.4104$ \\
\hline 0.8 & 0.8660 & 0.2038 & $>0.2474$ & 0.6736 & 0.3415 & $>0.4104$ \\
\hline 0.9 & 1.0264 & 0.2378 & $>0.2994$ & 0.7270 & 0.3762 & $>0.5579$ \\
\hline 1.0 & 1.1547 & 0.2665 & $>0.3464$ & 0.7698 & 0.4075 & $>0.6298$ \\
\hline 2.0 & 1.7320 & 0.4330 & $>0.6928$ & 0.9623 & 0.6186 & $>1.3324$ \\
\hline 3.0 & 1.9245 & 0.5145 & $>0.9279$ & 1.0264 & 0.7357 & $>2.0277$ \\
\hline 4.0 & 2.0207 & 0.5639 & $>1.1022$ & 1.0585 & 0.8107 & $>2.7218$ \\
\hline 5.0 & 2.0785 & 0.5973 & $>1.2372$ & 1.0777 & 0.8629 & $>3.4153$ \\
\hline 10 & 2.1394 & 0.6744 & $>1.6211$ & 1.1162 & 0.9888 & $>6.8808$ \\
\hline
\end{tabular}

\section{DISCUSSION :}

We present cosmological solution of a higher dimensional universe considering matter sector of the theory which arises from the extra dimensions. The evolution of the extra dimensions scale factor is described by Kasner-type behavior. The solutions 
obtained here is identified with the $(3+1)$ dimensional flat $(\mathrm{k}=0)$ universe with imperfect fluid which are described by Eckart, TIS and FIS theories. In the framework of imperfect viscous fluid the evolution of the early universe admits both power law inflation and power law slow expansion which is decided by the viscous behaviour of the imperfect fluid. The overall evolution of the internal space is contracting in nature whereas the 3 -physical space expands. For $\gamma=\frac{4}{3}$ one obtains solution with $m \geq \frac{1}{2}$ whereas for $\gamma=2$ we get solution for $m>\frac{1}{3}$. However, $m=\frac{1}{2}$ indicates non viscous universe for $\gamma=\frac{4}{3}$. Thus the usual radiation dominated behaviour of the universe $(a(t) \sim \sqrt{t})$ is obtained in this case. ${ }^{4}$ In the FIS theory we obtain the behaviour of the temperature of the universe during the regime. The temperature of the universe is determined in the FIS theory admits a decreaseing mode during the expansion of the 3-physical space determined by the viscous co-efficient $\beta$ in the theory. One interesting aspect of the solution is that for a given expansion of the universe $\beta$ is fixed in Eckart and TIS theories with values $\beta_{\text {Eckart }}>\beta_{\text {TIS }}$, however one gets a wide range of values of $\beta$ in FIS theories with lower bound least for $\gamma=\frac{4}{3}$.

Acknowledgement : I would like to thank the Inter-University Centre for Astronomy and Astrophysics ( IUCAA ) Pune for awarding a Visiting Associateship and providing a facility where this work was carried out. I am grateful to Professor N. Dadhich for his suggestion and cordial discussion. Finally, I like to thank Professor S. Mukherjee for providing IUCAA Reference Centre (IRC) facilities at North Bengal University for completing the work. 


\section{References}

[1] T. Kaluza, Sitz. Preuss. Acad. Wiss F 1, 966 (1921); O. Klein, A. Phys. 37, 895 (1926).

[2] A. Chodos and S. Detweiler, Phys. Rev. D 212167 (1980); Shafi Q. Shafi and C. Wetterich, Nucl. Phys. B 289 (1987), 787; C. Wetterich, Phys. Letts. B 113, 377 (1982); F. S. Accetta, M. Gleiser, R. Holman and E. W. Kolb, Nucl. Phys. B 276, 501 (1986) ; D. Lorentz Petzold, Prog. theor. Phys. B 78, 969 (1988); Mod. Phys. Lett. A 3827 (1988); B. C. Paul and S. Mukherjee, Phys. Rev. D 422595 (1990).

[3] P. Ponce de Leon, and P. S. Wesson, J. Math. Phys. 34, 4080 (1993) ; P. S. Wesson, J. Ponce de Leon, P. Lim and H. Liu, Int. J. Mod. Phys. D 2, 163 (1993); S. Chatterjee, Astron. Astrophys. 230, 1 (1990) ; H. Liu, and P. S. Wesson, Int. J. Mod. Phys. D 3, 627 (1994) ; H. Liu and P. S. Wesson, J. Math. Phys. 34, 4070 (1993).

[4] J. Ibanez and E. Vardaguer, Astrophys. J. 306, 401 (1986).

[5] R. J. Gleiser and M. C. Diaz, Phys. Rev. D 37, 3761 (1988).

[6] K. D. Krori, P. Borgohain, and K. Das, Gen. Rel. Grav. 22, 791 (1990).

[7] C. Eckert, Phys. Rev. 306, 401 (1986).

[8] W. A. Hiscock and L. Lindblom, Phys. Rev. D 31, 725 (1985).

[9] W. A. Hiscock, Phys. Rev. D 33, 1527 (1986). 
[10] D. Pavon, D. Jou. and J. Casas Va'zques, Ann. Inst. Henri Poincare' A 36 79 (1982) ; W. Israel, Phys. (NY) 100310 (1996); W. Israel and J. M. Stewart, Ann. Phys. (NY) 118341 (1970); R. Maarteen, Class. Quantum Grav. 12 1455 (1995) ; Hanno Rund Lectures (June 1996) ed. S. D. Mahraj.

[11] D. Jou, J. Casas Va'zques and L. Lebon, Extended Irreversible Thermodynamics ( Springer Verlag, Berlin 1993). 